\title{
CAPÍTULO 09: INFLUÊNCIA DAS CULTURAS DO AMENDOIM E DA CANA-DE- AÇÚCAR, NOS PROCESSOS DE PERDAS DE NITROGÊNIO, FÓSFORO, ÁGUA E SEDIMENTOS
}

\section{CAPÍTULO 09: INFLUENCIA DE LOS CULTIVOS DE MANÍ Y AZÚCAR EN LOS PROCESOS DE PÉRDIDA DE NITRÓGENO, FÓSFORO, AGUA Y SEDIMENTOS}

\author{
CHAPTER 09: INFLUENCE OF PEANUT AND SUGARCANE CROPS ON \\ NITROGEN, PHOSPHORUS, WATER AND SEDIMENT LOSS PROCESSES
}

\author{
Paulo Alexandre da Silva ${ }^{1}$; Teresa Cristina Tarlé Pissarra ${ }^{2}$; Maria Elisa Vicentini ${ }^{3}$; Alan Rodrigo Panosso ${ }^{4}$; \\ Glauco de Souza Rolim 5
}

DOI: https://doi.org/10.31692/978-65-88970-07-2.135-149

\begin{abstract}
RESUMO
O sistema de rotação de culturas da região de Sertãozinho-SP utiliza a cana-de-açúcar e o amendoim, fazendo com que este seja um dos principais produtores do mundo, mostrando que este sistema de produção é bastante benéfico, pois propicia o maior rendimento produtivo e financeiro, além de promover a recuperação dos solos e a diminuição das perdas de água, nutrientes e sedimentos. Objetivo: determinar os processos de perdas água, nitrogênio, fósforo e sedimentos, sob a influência do uso e ocupação do solo de dois sistemas de produção, amendoim e cana-de-açúcar, por meio do modelo SWAT, mantendo as características topográficas, os fatores físicos e climáticos da sub-bacia hidrográfica do Rio Mogi-Guaçu, no município de Sertãozinho - SP. A cultura influencia no balanço hidrológico e nas perdas de nutrientes e sedimentos. Além disso, existem diversas ferramentas que podem medir ou estimar esses processos. Pensando nisso, foram levantadas as seguintes hipóteses: Hipótese 1: A ferramenta Swat pode indicar as perdas de água, nutrientes e sedimentos em diferentes sistemas de produção; Hipótese 2: Os processos de perdas de água, nutrientes e sedimentos é maior para a cultura do amendoim com relação à cana-de-açúcar. O estudo foi realizado em Sertãozinho-SP. O modelo digital de elevação foi adquirido na base de dados mundial HydroSHEDS - USGS. Para o mapa de solos foi utilizado o modelo pedológico da Empresa Brasileira de Pesquisa Agropecuária. O mapa de uso e ocupação do solo foi obtido pelas junções das bandas do satélite Landsat- 8 . Os dados climáticos usados foram: precipitação, vento, umidade relativa e solar no formato de arquivo SWAT, no período de 1979 a 2014. Para a evapotranspiração real os valores foram maiores na cana-de-açúcar com relação ao amendoim (906,7 e $886,1 \mathrm{~mm}$, respectivamente). A quantidade de sedimentos foi maior no amendoim, quando comparado com a cana $(300,66$ e $280,69 \mathrm{~mm}$, respectivamente). As maiores perdas de nitrogênio e fósforo também foram maiores no amendoim com relação à cana (118.44 e $34.48 \mathrm{~kg} / \mathrm{ha}$ para $\mathrm{N} ; 5.456$ e $0.805 \mathrm{~kg} / \mathrm{ha}$ para $\mathrm{P}$, respectivamente). Conclui-se que a simulação com a cultura do amendoim foi a que registrou as maiores perdas de água, nutrientes e sedimentos, quando comparado com a cana, mostrando que o manejo e o uso do solo foram fatores que influenciaram neste processo. Assim, esses tipos de estudos podem servir nas tomadas de decisões com relação às políticas ambientais, colaborando com a conservação do solo e da água nas sub-bacias brasileiras.
\end{abstract}

Palavras-Chave: Cana-de-açúcar, amendoim, perdas de águas, perdas de nutrientes e sedimentos, Swat.

RESUMEN

\footnotetext{
1 M.Sc. em Agronomia, Universidade Estadual Paulista "Júlio de Mesquita Filho", paulo.a.silva@,

2 Professora Dra., Universidade Estadual Paulista "Júlio de Mesquita Filho", teresa.pissarra@unesp.br

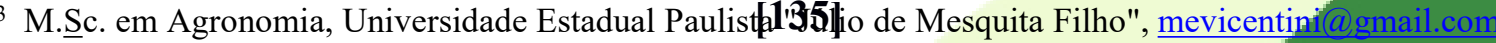

4 Professor Dr., Universidade Estadual Paulista "Júlio de Mesquita Filho", alan.panosso@unesp.br

5 Professor Dr., Universidade Estadual Paulista "Júlio de Mesquita Filho", glauco.rolim@unesp.br
} 
El sistema de rotación de cultivos en la región Sertãozinho-SP utiliza caña de azúcar y maní, lo que lo convierte en uno de los principales productores del mundo, lo que demuestra que este sistema de producción es bastante beneficioso, ya que brinda el mayor rendimiento. productiva y financiera, además de promover la recuperación de suelos y reducir las pérdidas de agua, nutrientes y sedimentos. Objetivo: determinar los procesos de pérdida de agua, nitrógeno, fósforo y sedimentos, bajo la influencia del uso y ocupación del suelo de dos sistemas de producción, maní y caña de azúcar, a través del modelo SWAT, manteniendo las características topográficas, la factores físicos y climáticos de la subcuenca del río Mogi-Guaçu, en el municipio de Sertãozinho - SP. El cultivo influye en el equilibrio hidrológico y la pérdida de nutrientes y sedimentos. Además, existen varias herramientas que pueden medir o estimar estos procesos. Con esto en mente, se plantearon las siguientes hipótesis: Hipótesis 1: La herramienta Swat puede indicar pérdidas de agua, nutrientes y sedimentos en diferentes sistemas de producción; Hipótesis 2: Los procesos de pérdida de agua, nutrientes y sedimentos son mayores para el cultivo de maní en relación a la caña de azúcar. El estudio se llevó a cabo en Sertãozinho-SP. El modelo de elevación digital se adquirió en la base de datos mundial HydroSHEDS - USGS. Para el mapa de suelos se utilizó el modelo pedológico de la Corporación Brasileña de Investigaciones Agropecuarias. El mapa de uso y ocupación del suelo se obtuvo uniendo las bandas del satélite Landsat-8. Los datos climáticos utilizados fueron: precipitación, viento, humedad relativa y solar en el formato de archivo SWAT, de 1979 a 2014. Para la evapotranspiración real los valores fueron mayores en la caña de azúcar en relación al maní $(906,7$ y 886,1 mm, respectivamente). La cantidad de sedimento fue mayor en el maní, en comparación con la caña de azúcar (300,66 y 280,69 mm, respectivamente). Las mayores pérdidas de nitrógeno y fósforo también fueron mayores en el maní en relación a la caña de azúcar (118.44 y $34.48 \mathrm{~kg}$ / ha para N; 5.456 y $0.805 \mathrm{~kg}$ / ha para $\mathrm{P}$, respectivamente). Se concluye que la simulación con el cultivo de maní fue la que registró las mayores pérdidas de agua, nutrientes y sedimentos, al compararse con la caña, demostrando que el manejo y el uso del suelo fueron factores que incidieron en este proceso. Así, este tipo de estudios pueden servir en la toma de decisiones en materia de políticas ambientales, colaborando con la conservación del suelo y el agua en las subcuencas brasileñas.

Palabras Clave: Caña de azúcar, maní, pérdidas de agua, pérdidas de nutrientes y sedimentos, Swat.

\begin{abstract}
The crop rotation system in the Sertãozinho-SP region uses sugar cane and peanuts, making it one of the main producers in the world, showing that this production system is quite beneficial, as it provides the highest yield productive and financial, in addition to promoting soil recovery and reducing water, nutrient and sediment losses. Objective: to determine the processes of loss of water, nitrogen, phosphorus and sediments, under the influence of the use and occupation of the soil of two production systems, peanuts and sugarcane, through the SWAT model, maintaining the topographic characteristics, the physical and climatic factors of the Mogi-Guaçu River sub-basin, in the municipality of Sertãozinho - SP. The culture influences the hydrological balance and the loss of nutrients and sediments. In addition, there are several tools that can measure or estimate these processes. With this in mind, the following hypotheses were raised: Hypothesis 1: The Swat tool can indicate losses of water, nutrients and sediments in different production systems; Hypothesis 2: The processes of loss of water, nutrients and sediment is greater for the cultivation of peanuts in relation to sugarcane. The study was carried out in Sertãozinho-SP. The digital elevation model was acquired in the worldwide HydroSHEDS - USGS database. For the soil map, the pedological model of the Brazilian Agricultural Research Corporation was used. The land use and occupation map was obtained by joining the bands of the Landsat- 8 satellite. The climatic data used were: precipitation, wind, relative humidity and solar in the SWAT file format, from 1979 to 2014. For real evapotranspiration the values were higher in sugarcane in relation to peanuts ( 906.7 and $886.1 \mathrm{~mm}$, respectively). The amount of sediment was higher in peanuts, when compared to sugarcane $(300.66$ and $280.69 \mathrm{~mm}$, respectively). The highest losses of nitrogen and phosphorus were also greater in peanuts in relation to sugarcane (118.44 and $34.48 \mathrm{~kg}$ / ha for N; 5,456 $\$ 3369.805 \mathrm{~kg}$ / ha for P, respectively). It is concluded that the simulation with the peanut culture was the one that registered the biggest losses of water, nutrients and sediments, when compared with the cane, showing that the management and the use of
\end{abstract}


the soil were factors that influenced in this process. Thus, these types of studies can serve in decisionmaking in relation to environmental policies, collaborating with the conservation of soil and water in Brazilian sub-basins.

Keywords: Sugarcane, peanuts, water losses, nutrient and sediment losses, Swat.

\section{INTRODUÇÃO}

O Brasil é o maior produtor de cana-de-açúcar (Saccharum spp.) do mundo. Segundo os dados da Companhia Nacional de Abastecimento - CONAB, foram produzidos cerca de 615,84 milhões de toneladas de cana-de-açúcar em 8,63 milhões de hectares na safra 2018/2019. O estado de São Paulo foi o maior produtor do país, com 5,2 milhões de hectares e produção de 334,52 milhões de toneladas, compreendendo $52 \%$ da cana processada (CONAB, 2019), sendo a região da Alta Mogiana (Ribeirão Preto, Dumont, Jaboticabal e Sertãozinho) uma das grandes produtoras do estado.

Após as colheitas consecutivas da cana-de-açúcar tem-se o término do ciclo da cultura. Devido ao grande tempo de permanência da cultura em uma mesma área, o solo tende a perder as características agronômicas que propiciam o desenvolvimento das plantas. Uma forma de propiciar a recuperação deste solo é a rotação das culturas. A rotação de culturas é uma técnica que promove a alternância anual das espécies vegetais em uma mesma área de cultivo agrícola, onde as plantas escolhidas apresentam propósitos comerciais e possuem a capacidade de recuperação do solo. As principais vantagens desse sistema são: a diversidade da produção de alimentos e produtos agrícolas, melhorias nas características físicas, químicas e biológicas do solo, reposição da matéria orgânica, proteção do solo e ajuda no controle de plantas daninhas, doenças e pragas. As operações agrícolas envolvidas no processo de rotação são: retirada da cana (entre setembro e outubro), destruição da soqueira, calagem, preparo do solo, plantio da cultura anual, colheita (entre fevereiro e março) e novo plantio de cana logo em seguida. Geralmente, as reformas dos canaviais são feitas por espécies chamadas de adubos verdes, que possuem como características principais a formação de uma cobertura superficial e a manutenção das propriedades físicas, químicas e biológicas do solo, inclusive em profundidade. As plantas mais utilizadas são: Crotalaria juncea, soja e amendoim. (BOLONHEZI e PEREIRA, 1999).

Na cidade de Sertãozinho - SP, a principal cultura utilizada para a rotação é o amendoim. Segundo os dados da Companhia Nacional de Abastecimento, a produção total brasileira de amendoim na safra 2017/18 foi de 515,9 mil toneladas, em 139,3 mil hectares, 
com produtividade média de $3.704 \mathrm{~kg} / \mathrm{ha}$ (CONAB, 2018). A grande maioria dos amendoinzais nacionais ficam localizados dentro do Estado de São Paulo que detém cerca de 93,8\% da produção nacional. Os principais pólos de cultivo são as regiões da Alta Mogiana (Ribeirão Preto, Dumont, Jaboticabal e Sertãozinho) e Alta Paulista (Tupã e Marília). Boa parte são produtores canavieiros que plantam o amendoim em rotação com a cana-de-açúcar. Estimativas mostram que $90 \%$ do cultivo dessa leguminosa pertence a esses canavicultores. Além disso, nestas mesmas regiões estão localizadas as maiores indústrias de produtos à base de amendoim brasileira, fazendo com que estas sejam reconhecidas como as maiores produtoras de amendoim do Brasil.

A colheita mecanizada da cana-de-açúcar é uma prática conservacionista, visto que a palhada permanece na superfície do solo, diminuindo a sua erosão, além de evitar a emissão de dióxido de carbono, ocasionada pelas queimadas. Furlani Neto (1994) ao comparar cana crua e cana queimada destacou como vantagens a maior proteção do solo contra erosão, a redução do uso de herbicidas, a melhor matéria-prima para indústria, a maior incorporação de matéria orgânica ao solo, a maior atividade microbiana do solo e redução da poluição ambiental. Martins Filho (2007) em seus estudos diz que quando os resíduos vegetais são deixados ou mantidos em contato direto com a superfície do solo, são extremamente eficientes em controlar o processo de erosão em entressulcos.

O objetivo deste trabalho foi determinar os processos de perdas de solo, água e sedimentos, sob a influência do uso, ocupação do solo e da paisagem de dois sistemas de produção, amendoim (leguminosa) e cana-de-açúcar (gramínea), por meio do modelo SWAT, no programa de mapeamento digital ArcGis, mantendo as características topográficas e os fatores físicos e climáticos da sub-bacia hidrográfica do Rio Mogi-Guaçu, localizada no município de Sertãozinho - SP.

\section{FUNDAMENTAÇÃO TEÓRICA}

Essa parte do artigo servirá como uma base teórica para os temas abordados no trabalho, bem como, os principais elementos e conceitos usados nesse estudo. Ao final serão apresentadas as hipóteses testadas na pesquisa.

Para o entendimento do funcionamento do balanço hídrico, os estudos hidrológicos realizados em bacias hidrográficas são ${ }_{[\mathbf{1 3 8}}$ grande importância, pois propiciam o compreendimento dos processos que controlam o movimento da água e seus possíveis 
quantificações no sistema, solo-planta-atmosfera.

Com a crescente demanda dos Sistemas de Informações Geográficas (SIG), os modelos hidrológicos vem sendo aplicados e desenvolvidos com foco nas características físicas do estudo, visto que o uso de um SIG pode auxiliar no fornecimento dos parâmetros utilizados nos diferentes modelos (VEITH et al., 2010). Vieux (2004) mostrou que a integração da hidrologia no SIG foi capaz de capturar, armazenar, manipular, analisar e visualizar diversos grupos de dados georreferenciados e a hidrologia proporcionou o aumento do conhecimento espacial nas áreas estudadas, porém para as melhores aplicações dos modelos hidrológicos são necessárias grandes quantidades de dados. Os modelos tentam representar o movimento natural da água, por meio dos dados calibrados, onde o processo de calibração é de grande importância, pois isso resulta na diminuição dos erros entre a saída do modelo e os dados observados, como muitos parâmetros não medidos isso faz com que a precisão desses dados estimados seja dificultada (VEITH et al., 2010).

O modelo SWAT (Soil and Water Assessment Tool) estuda a bacia hidrográfica por meio do tempo contínuo, analisando em uma escala diária. Os idealizadores tiveram como objetivo desenvolver um modelo para mensurar e avaliar os efeitos das mudanças e do uso do solo com relação aos recursos hídricos nas bacias hidrográficas rurais não monitoradas (Arnold et al., 1998). O SWAT é basicamente a aplicação da experiência na área da modelagem que a USDA-ARS obteve no decorrer dos mais de 30 anos de pesquisa (GASSMAN et al., 2007). Já foram registrados mais de 70 trabalhos que utilizaram a aplicação do modelo, nas mais diversas regiões brasileiras (GARBOSSA et al. 2011), mostrando estudos que compararam a eficiência dos modelos frente à simulação da vazão, sedimentos e nutrientes. Im et al. (2003) compararam os resultados obtidos entre os modelos HSPF (Hydrologic Simulation Program-Fortran) (BICKNELL et al., 1996) e o SWAT na bacia do córrego Pelecat, de 12048 hectares, no Estado da Virgínia (EUA) e observaram que os dois modelos simularam com exceto a vazão, sedimentos e nutrientes no período de simulação. Analisando as discrepâncias das tendências de cargas mensais e as cargas anuais, o modelo HSPF estimou a hidrologia e as características da qualidade da água de maneira mais precisa do que SWAT em todos os os pontos estudados dentro da bacia hidrográfica. Entretanto, os autores dizem que o modelo HSPF é mais complexo do que o modelo SWAT, devido a quantidade de parâmetros usados para estimar o ciclo hidrológico e o transporte de sedimentos e nutrientes. 
Os processos hidrossedimentológicos são resultantes das junções dos processos hidrológicos e sedimentológicos. A água é um dos principais agentes do intemperismo, resultando nos processos de desagregação, erosão, transporte, sedimentação, depósito e consolidação nas bacias hidrográficas. Segundo Pereira (2003) quando ocorre o desbalanço do equilíbrio no solo, devido a força oriundas da erosão hídrica, observa-se que as características do terreno podem interferir na intensidade do processo erosivo. Sendo assim, as condições mais importantes são: a declividade do terreno, capacidade de infiltração da água no solo, à distância percorrida pelo escoamento superficial, a rugosidade superficial, a resistência do solo à ação erosiva da chuva e a percentagem de cobertura do solo existente à época da ocorrência da chuva. Outro fator que deve ser considerado é o tipo da cultura, pois a mesma pode influenciar no balanço hidrológico e nas perdas de nutrientes e sedimentos. Diversos estudos são realizados e existem diversas ferramentas que podem medir ou estimar esses processos. Pensando nisso, foram levantadas as seguintes hipóteses:

Hipótese 1 = A ferramenta SWAT pode indicar as perdas de água, nutrientes e sedimentos em diferentes sistemas de produção;

Hipótese 2 = O processo de perdas de água, nutrientes e sedimentos é maior para a cultura do amendoim com relação à cana-de-açúcar.

\section{METODOLOGIA}

\section{Área de estudo}

O Município de Sertãozinho está localizado na região Centro-Norte do Estado de São Paulo, situado no Planalto Ocidental Paulista, sob as coordenadas geográficas $21^{\circ} 08^{\prime} 16^{\prime \prime}$ Latitude Sul e $47^{\circ} 59^{\prime}$ 25" Longitude Oeste de Greenwich. Apresenta área territorial de aproximadamente 403,89 $\mathrm{km}^{2}$ (IBGE, 2010).

Segundo a classificação de Köppen, o clima é do tipo Aw (tropical úmido e seco). A temperatura média anual na região é de $22,8^{\circ} \mathrm{C}$, a precipitação média anual está ao redor de 1.588,5 mm (CEPAGRI). O relevo predominante é o suave-ondulado e ondulado, com altitude variando de $310 \mathrm{~m}$ a $610 \mathrm{~m}$. O Município está vinculado a ABAGH/RP (Associação Brasileira do Agronegócio da Região de Ribeirão Preto) e faz parte da UGRHI - da Bacia Mogi-Guaçu, com parte de sua área na UGRHI - (Rio Pardo) (ABAGH/RP) e também está localizada sob a região da alta Mogiana. [1400] relação ao bioma, a cobertura vegetal predominante é a de Cerrado (IBGE, 2010). O município foi bastante desmatado devido ao 
avanço do agronegócio, por meio da agropecuária (cultura de cana de açúcar, a produção de leite e laticínios) e agroindústrias, sobrando praticamente algumas áreas de reservas de mata nativa.

\section{Dados utilizados no ArcSWAT}

O modelo digital de elevação (MDE) para a área de estudo foi adquirido por meio da base de dados mundial HydroSHEDS - USGS, que possui uma resolução aproximada de 1 quilômetro. A Figura 1 mostra o MDE para o município de Sertãozinho - SP, com destaque em vermelho para a sub-bacia em estudo.

Figura 1: Mapa Digital de elevação digital da cidade de Sertãozinho - SP. Demarcação da sub-bacia de estudo da bacia de Mogi Guaçu (vermelho).

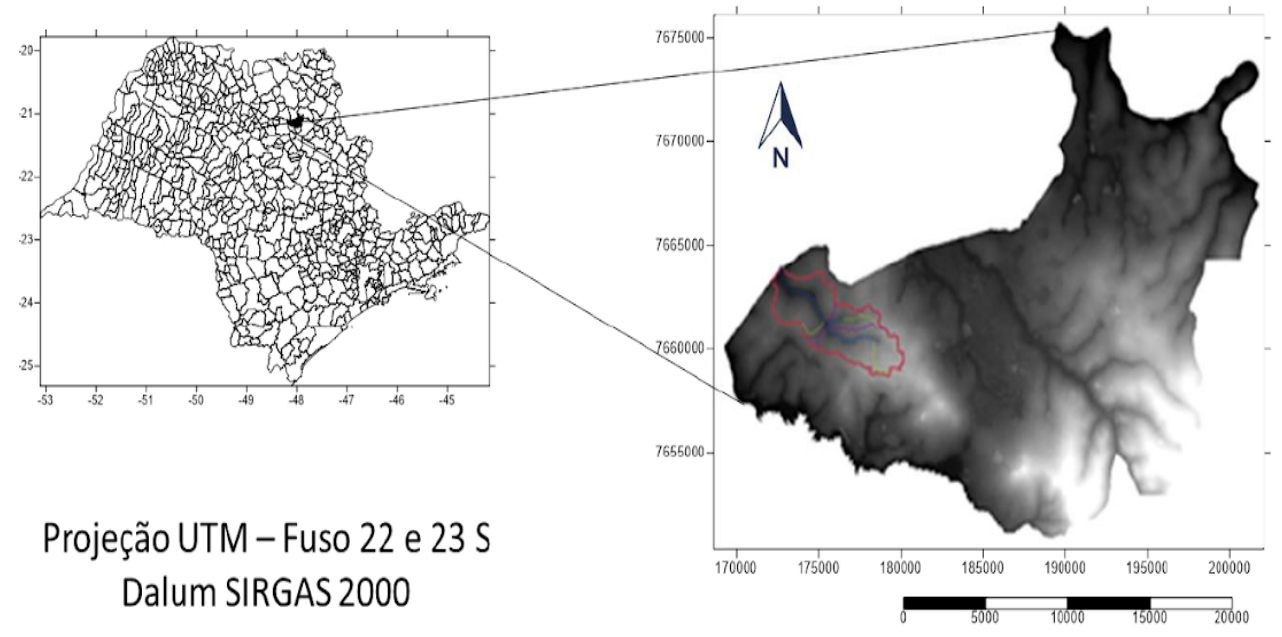

Fonte: Própria (2020).

Para a elaboração do mapa de solos foi utilizado o modelo pedológico disponibilizado pela Empresa Brasileira de Pesquisa Agropecuária (EMBRAPA, 1999), que tem como base o sistema brasileiro de classificação de solos (IBGE, 2001).

O mapa de uso e ocupação do solo foi obtido pelas junções das bandas fornecidas pelo satélite Landsat-8. Lançado em fevereiro de 2013. O satélite Landsat-8 é um sensor que apresenta órbita praticamente polar, posicionando-se de maneira heliossíncrona a uma altitude de aproximadamente $705 \mathrm{~km}$. A resolução temporal utilizada foi de 16 dias e a espacial foi de 30 metros para as bandas do visível.

Os dados climáticos foram retirados do sistema dos Centros Nacionais para Previsão Ambiental (NCEP), que pertence a Reanálise do Sistema de Previsão Climática (CFSR), onde foram coletados os dados de precipitação, vento, umidade relativa e solar no formato de arquivo SWAT, no período de 1979 a 2014. [0\$1\$doftwares utilizados foram o ArcSWAT, em conjunto com o ArcGIS 10.3 para as análises hidrológicas e o de perdas de sedimentos. 


\section{RESULTADOS E DISCUSSÃO}

Na Tabela 1 estão listados os coeficientes hidrológicos oferecidos pela simulação do programa Swat para as culturas de cana-de-açúcar e amendoim. Os parâmetros Evapotranspiração potencial (PET), precipitação, e a ascensão capilar permaneceram constantes em ambas as áreas de estudo, mostrando que o uso e a ocupação do solo não influenciaram nessas características simuladas, sendo necessário a calibração dessas informações para o modelo. Ferreira e Uagoda (2007) estudaram o ciclo hidrológico e observaram a superestimação do fluxo de base e do escoamento superficial para as condições de cerrado, mostrando a necessidade de calibração para esse modelo.

Tabela 1. Perdas hídricas da sub-bacia do rio Mogi-Guaçu através do modelo SWAT.

\begin{tabular}{ccc}
\hline Parâmetros & Cana (mm) & Amendoim (mm) \\
\hline Evapotranspiração potencial & 1573,30 & 1573,30 \\
Evapotranspiração real & 906,70 & 886,10 \\
Precipitação & 1667,40 & 1667,40 \\
Escoamento superficial & 280,69 & 300,66 \\
Fluxo lateral & 12,59 & 8,15 \\
Percolação da água para o aquífero raso & 467,79 & 472,03 \\
Retorno do fluxo & 412,92 & 416,95 \\
Ascensão capilar & 31,47 & 31,47 \\
Recarga para o aquífero profundo & 23,39 & 23,60 \\
Média da produção de sedimentos de terras altas $\mathbf{( M g / h a )}$ & 0,08 & 19.12 \\
\hline
\end{tabular}

Fonte: Própria (2020).

No estudo foi observado que as culturas influenciaram na evapotranspiração real e no escoamento superficial. Para a cana-de-açúcar foram observados os valores de 906,7 mm e $280,69 \mathrm{~mm}$, já para a cultura do amendoim foram $886,1 \mathrm{~mm}$ e $300,66 \mathrm{~mm}$, respectivamente. Isto mostra que a cana por produzir maior biomassa faz com que o processo de evapotranspiração real seja maior, quando comparado com a cultura do amendoim. Porém quando há o escoamento superficial da água do solo no amendoim esse processo é maior, pois devido à ausência de palha na superfície, o solo acaba saturando mais rápido, aumento o fluxo de água e consequentemente, a percolação de sedimentos. Yan et al. (2013) observaram que a alteração no uso do solo provoca variação do escoamento superficial de uma bacia hidrográfica, fato ocasionado principalmente pela evapotranspiração e condutividade hidráulica.

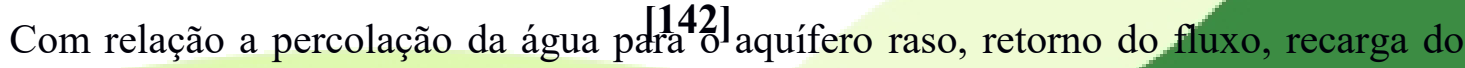


aquífero profundo e a média da produção de sedimentos do planalto foram observados os maiores índices para a cultura amendoim. Para o amendoim os valores encontrados foram $472,03,416,95,23,6$ e 19,12 mm, enquanto que para a cana foram 467,79, 412,92, 23,39 e $0,08 \mathrm{~mm}$, respectivamente. Dessa forma é possível observar que a presença da palhada no solo auxilia no equilíbrio hidrológico do solo, diminuindo a velocidade do fluxo de nas camadas profundas e superficiais. Borges et al. (2014) avaliaram os efeitos de diferentes tipos de cobertura na manutenção da umidade do solo, sob condição de chuva natural na cultura do milho (Zea mays L.), em regime de sequeiro e observaram que o cultivo do milho em nível com barramento em pedras, associado com cobertura morta ou com cordão vegetativo de palma forrageira atua eficientemente na redução das perdas de água, quando comparado ao solo descoberto, contribuindo para melhor aproveitamento da água da chuva e maior produtividade de grãos.

\section{Balanço do Nitrogênio}

No estudo das perdas de nitrogênio no solo, sob os diferentes sistemas de produção foram observados que as culturas influenciaram diretamente na estimativa da quantidade e na forma de como o nitrogênio foi perdido. De maneira geral, observa-se que o sistema de produção da cultura do amendoim foi o que apresentou a maior perda de nitrogênio segundo a simulação, totalizando $118,44 \mathrm{~kg} / \mathrm{ha}$, já na cultura da cana-de-açúcar esta perda foi de 34,48 $\mathrm{kg} / \mathrm{ha}$. Este fato aconteceu devido o cultivo de amendoim necessitar de muito mais nitrogênio, quando comparado a cana, resultando também na maior perda desse nutriente. As leguminosas são muito mais exigentes, visto que o sistema radicular superficial e ciclo são mais curtos e que o nitrogênio é absorvido em quantidade mais elevada. Estudos apontam que oleaginosas apresentaram deficiências de $\mathrm{N}$, quando cultivado em sistema plantio direto, sendo necessário maiores doses (SORATTO et al. 2004), principalmente quando cultivado em sucessão a gramíneas (GOMES JÚNIOR et al. 2008).

Para as perdas provocadas pelo escoamento superficial, lixiviação, fluxo lateral e as águas subterrâneas do nitrato, quando comparada com a quantidade total foram maiores na cultura da cana, apresentando as seguintes quantidades perdidas: $0,46 \mathrm{~kg} / \mathrm{ha}(1,3 \%), 32,74$ $\mathrm{kg} / \mathrm{ha}(95 \%), 0,19 \mathrm{~kg} / \mathrm{ha}(0,5 \%)$ e $0,69 \mathrm{~kg} / \mathrm{ha}(2 \%)$, respectivamente. Já para a cultura do amendoim, as perdas foram de $0,34 \mathrm{~kg} / \mathrm{ha}(0,3 \%), 85,99 \mathrm{~kg} / \mathrm{ha}(72,6 \%), 0,37 \mathrm{~kg} / \mathrm{ha}(0,3 \%) \mathrm{e}$ $1,84 \mathrm{~kg} / \mathrm{ha}(1,6 \%)$, respectivamente (Tabela 4$) .43]$ 
Tabela 2. Estimativa das perdas de nitrogênio na sub-bacia do rio Mogi-Guaçu.

\begin{tabular}{ccccc}
\hline Parâmetros & $\begin{array}{c}\text { Cana-de-açúcar } \\
(\mathbf{k g} / \mathbf{h a})\end{array}$ & $\begin{array}{c}\text { Porcentagem } \\
\mathbf{( \% )}\end{array}$ & $\begin{array}{c}\text { Amendoim } \\
(\mathbf{k g} / \mathbf{h a})\end{array}$ & Porcentagem (\%) \\
\hline $\begin{array}{c}\text { Perda total de } \\
\text { nitrogênio }\end{array}$ & 34,48 & 100 & 118,44 & 100 \\
$\begin{array}{c}\text { Nitrogênio orgânico } \\
\text { Escoamento superficial }\end{array}$ & 0,40 & 1,20 & 29,90 & 25,20 \\
$\quad$ de nitrato & 0,46 & 1,30 & 0,34 & 0,30 \\
Nitrato lixiviado & 32,74 & 95,00 & 85,99 & 72,60 \\
$\begin{array}{c}\text { Fluxo lateral de nitrato } \\
\text { Nitrato de águas } \\
\text { subterrânea }\end{array}$ & 0,19 & 0,50 & 0,37 & 0,30 \\
\hline
\end{tabular}

Fonte: Própria (2020).

Nos dois sistemas de produção observou-se que a principal forma de perda de nitrogênio foi por meio da lixiviação, sendo esse processo mais agressivo sob o cultivo da cana. Esse processo colaborou com o acúmulo do nitrogênio em águas subterrâneas, sendo um outro indicativo de perda. A lixiviação de nitrato é influenciada diretamente pelos fatores que determinam o fluxo de água no solo e pela concentração de $\mathrm{NO}_{3}{ }^{-}$na solução (WHITE, 1987). Brum (1975) observou que as maiores concentrações de $\mathrm{NO}_{3}{ }^{-}$em profundidades inferiores acontecem porque geralmente $\mathrm{o} \mathrm{NH}_{4}{ }^{+}$são adsorvidos pelas cargas negativas do solo. Já o $\mathrm{NO}_{3}{ }^{-}$tende a ser lixiviado e acumulado na região das águas subterrâneas.

Com relação às perdas do nitrogênio orgânico, foi observado que esse processo foi maior na cultura do amendoim, totalizando $29,90 \mathrm{~kg} / \mathrm{ha}$, representando $25,2 \%$ da perda total. Já para a cultura da cana a quantidade perdida foi de $0,4 \mathrm{~kg} / \mathrm{ha}$, representando $1,2 \%$ da perda total. Devido às características dos sistemas de produção no amendoim, tem-se a maior mineralização do $\mathrm{N}$ orgânico por causa da atividade microbiana, facilitando a perda dessa fonte, quando comparado com a cana. Rosolem et al. (1990) observaram que houve o aumento da absorção de $\mathrm{N}$ devido a maior de maior mineralização de $\mathrm{N}$ orgânico do solo devido à elevação do $\mathrm{pH}$, da melhor distribuição do sistema radicular e também pela maior eficiência da fixação simbiótica do $\mathrm{N}_{2}$ em decorrência da menor acidez do solo (BLAMEY; CHAPMAN, 1982).

\section{Balanço do Fósforo}

No estudo das perdas de fósforo no solo, sob os diferentes sistemas de produção foram observados que as culturas influenciaram diretapqpnte na estimativa da quantidade e da forma de como o fósforo foi perdido. De maneira geral, observa-se que o sistema de produção da 
cultura do amendoim foi o que apresentou a maior perda de fósforo segundo a simulação, totalizando 5,46 kg/ha, já na cultura da cana-de-açúcar esta perda foi de 0,805 kg/ha. Devido a exposição do solo, a erosão hídrica atua de forma mais expressiva no amendoim, conforme discutido acima, resultando na maior perda de fósforo na área. Bertol (1994) verificou que as perdas totais de $\mathrm{P}$ foram maiores no solo sem cultivo (descoberto) do que no preparo convencional, cultivo mínimo e semeadura direta. A principal razão para esse comportamento, no caso do $\mathrm{P}$, foi a variação nas perdas de solo e água entre os tratamentos estudados, já que as concentrações do elemento foram maiores na semeadura direta do que nos demais tratamentos, especialmente nos sedimentos (Tabela 3).

Tabela 3. Estimativa das perdas de fósforo na sub-bacia do rio Mogi-Guaçu.

\begin{tabular}{ccccc}
\hline Índices de Perda & $\begin{array}{c}\text { Cana-de-açúcar } \\
(\mathbf{k g} / \mathbf{h a})\end{array}$ & $\begin{array}{c}\text { Porcentagem } \\
\mathbf{( \% )}\end{array}$ & $\begin{array}{c}\text { Amendoim } \\
(\mathbf{k g} / \mathbf{h a})\end{array}$ & $\begin{array}{c}\text { Porcentagem } \\
\mathbf{( \% )}\end{array}$ \\
\hline Perda total de fósforo & 0,805 & 100 & 5,456 & 100 \\
fósforo orgânico & 0,113 & 14,0 & 5,205 & 95,4 \\
$\begin{array}{c}\text { Escoamento superficial } \\
\text { de fósforo solúvel }\end{array}$ & 0,692 & 86,0 & 0,251 & 4,6 \\
\hline
\end{tabular}

Fonte: Própria (2020).

Com relação às perdas do fósforo orgânico foi observado que esse processo foi maior na cultura do amendoim, totalizando 5,205 kg/ha, representando $95,4 \%$ da perda total. Já para na cultura da cana, a quantidade perdida foi de $0,113 \mathrm{~kg} / \mathrm{ha}$, representando $14 \%$ da perda total. Devido às características dos sistemas de produção, no amendoim tem-se uma grande exposição do solo à mineralização, sobrando as formas menos lábeis, mostrando que a forma e a dinâmica do P no solo oscilam muito quanto ao uso da terra, por meio das mudanças na cobertura vegetal, na produção de biomassa e na ciclagem de nutrientes (MAGID et al., 1996).

Com relação ao escoamento superficial do fósforo solúvel foi observado que a maior quantidade e relação da perda de fósforo solúvel foi na cultura da cana, apresentando uma perda de $0,692 \mathrm{~kg} / \mathrm{ha}(86 \%)$, já o amendoim foi de $0,251 \mathrm{~kg} / \mathrm{ha}(4,6 \%)$. De maneira geral, o fósforo é pouco móvel no solo. A decomposição da palhada e a ação dos microrganismos na cana, promove reações sobre esses fósforos, que juntamente com a umidade, ocasionam maior escoamento superficial na cana, quando comparado ao amendoim. Novamente o sistema de produção interfere nessas perdas. A alta quantidade de matéria orgânica no solo e a microbiota na cultura da cana é muito mais desenvolvida que no amendoim, fazendo com que a mineralização do P seja mais intensa e evitando assim a adsorção dos fosfato $\mathbf{1} 1 \mathbf{4 5}$ bolo. Souza et al., (2006) observou que a ação da matéria orgânica diminuiu a adsorção dos íons fosfatos, atribuindo isso ao bloqueio dos sítios 
de adsorção. Traina et al. (1986) relataram que ácidos orgânicos (substâncias húmicas) da matéria orgânica podem solubilizar fosfatos de ferro e alumínio, reduzindo a precipitação de fosfatos com íons $\mathrm{Fe}$ e $\mathrm{Al}$ e diminuir a adsorção de fósforo por oxidróxidos de ferro e de alumínio, podendo adsorver as substâncias húmicas com grande energia e assim competirem com o fosfato pelos mesmos sítios de adsorção (BHATTI et al., 1998).

\section{CONCLUSÕES}

Conclui-se que a ferramenta SWAT foi capaz de estimar e diferenciar as potenciais perdas de água, sedimentos, nitrogênio e fósforo nas simulações em estudo, mostrando que o manejo, o uso, a ocupação e a paisagem do solo são os principais fatores que influenciam neste processo. Conforme a simulação, foram observadas as maiores perdas no sistema de produção da cultura do amendoim. As características hidrológicas e os manejos culturais foram os principais responsáveis pelas perdas de nitrogênio, fósforo e água.

Para a maior confiabilidade e precisão dos resultados simulados, precisa-se fazer as devidas calibrações, pois só assim serão obtidos os dados mais próximos da realidade, para assim esses tipos de estudos poderem servir nas tomadas de decisões com relação às políticas ambientais, colaborando com a conservação do solo e da água nas sub-bacias brasileiras.

\section{REFERÊNCIAS}

ABAG/RP, Associação Brasileira do Agronegócio da Região de Ribeirão Preto.

Disponível em http://www.abagrp.org.br. Acesso em 18 de jun. 2019.

ARNOLD, J. G.; SRINIVASAN, R. MUTTIAH, R. S. WILLIAMS, J. R. Large area hydrologic modeling and assessment part I: Model development. Journal of the American Water Resources Association, v. 34, n. 1, p. 1-17, 1998.

BERTOL, I. Erosão hídrica em Cambissolo Húmico distrófico sob diferentes preparos do solo e rotação de culturas. Revista Brasileira de Ciência do Solo, Viçosa, v. 18, n. 2, p. 267-271, 1994.

BHATTI, J.S.; COMERFORD, N.B.; JOHNSTON, C.T. Influence of oxalate and soil organic matter on sorption and desorption of phosphate onto a Spodic horizon. Soil Science Society American Journal, Madison, v. 62, p.1089-1095, 1998.

BICKNELL, B. R.; IMHOFF, J. C.; KITTLE JR, J. L.; DONIGIAN JR, A. S.; JOHANSON, R. C. Hydrological simulation program-FORTRAN. user's manual for release 11. US EPA, 1996. 
BLAMEY, F. P. C.; CHAPMAN, J. Soil ameriolation effects on peanut growth, yield and quality. Plant and Soil, The Hague, v. 65, p. 319-334, 1982.

BOLONHEZI, D.; PEREIRA, J. C. V. N. A. Plantio direto na Alta Mogiana. O Agronômico, Campinas, v. 51, p. 12-15, 1999.

BORGES, T.K.S.; MONTENEGRO, A.A.A.; SANTOS, T.E.M.; SILVA,D.D.; SILVA JUNIOR. V.P. Influência de práticas conservacionistas na umidade do solo e no cultivo do milho (Zea mays L.) em semiárido nordestino. Revista Brasileira de Ciência do Solo, v. 38, p. 1862-1873, 2014.

BRUM, A. C. R. Efeito de manejo e exploração agrícola na densidade populacional de bactérias nitrificadoras, em solo da unidade de mapeamento Santo Ãngelo. Santa Maria, 1975, 64p. Dissertação de Mestrado do Programa de Pós-Graduação em Agronomia, Universidade Federal de Santa Maria. 1975.

CEPAGRI. Centro de Pesquisas Meteorológicas e Climáticas Aplicadas a Agricultura. Disponível em <www.cepagri.unicamp.br>Acesso em 27 de jun. 2019.

CONAB (2018). Acompanhamento da safra brasileira: grãos, segundo a Companhia Nacional de Abastecimento, Brasília. Disponível em $<$ https://www.conab.gov.br/infoagro/safras/graos>. Acesso em: 27 jun. 2019.

CONAB (2019). Acompanhamento da safra brasileira: cana-de-açúcar, segundo a Companhia Nacional de Abastecimento, Brasília. Disponível em $<$ https://www.conab.gov.br/info-agro/safras/cana>. Acesso em: 27 jun. 2019.

EMBRAPA. Centro Nacional de Pesquisa de Solos. Sistema Brasileiro de Classificação de Solos. Rio de Janeiro, 412 p. 1999.

FERREIRA, R.S.; UAGODA, R.E.S. Análise da predição do balanço hídrico da bacia do ribeirão do Gama-DF através do modelo SWAT. Revista Brasileira de Geografia Física,v. 10, p. 880-893, 2017.

FURLANI NETO, V.L. Colheita mecanizada da cana-de-açúcar, STAB, Piracicaba, v.12, n.13, p.8-9, 1994.

GARBOSSA, L.H.P.; VASCONCELOS, L.R.C.; LAPA, K.R.; BLAINSKI, E.; PINHEIRO, A. The use and results of the Soil and Water Assessment Tool 137 in Brazil: A review from 1999 until 2010. 2011 International SWAT Conference \& Workshops, Toledo - Espanha, p. 27, 2011.

GASSMAN, P. W.; REYES, M. R.; GREEN, C. H.; ARNOLD, J. G. The soil and water assessment tool: historical development, applications, and future research directions.

Transactions of the ASABE, v. 50, n. 4, p. 1211-1250, 2007.

GOMES JÚNIOR, F. G.; SÁ, M.E.; VALÉRIQ4ł†Y.V. Filho. Nitrogênio no feijoeiro em sistema de plantio direto sobre gramíneas. Acta Scientiarum Agronomy, Maringá, v. 30, n. 3 , p. 387-395, 2008. 
IBGE, Instituto Brasileiro de Geografia e Estatística. Censo Populacional. 2010.

Disponível em www.ibge.gov.br. Acesso em 27 de jun. 2019.

IBGE - EMBRAPA - Mapa de Solos do Brasil. Rio de Janeiro: IBGE, 2001 - Escala 1:5.000.000. Acesso em 27 de jun. 2019.

IM, S.; BRANNAN, K.; MOSTAGHIMI, S.; CHO, J. A Comparison of SWAT and HSPF Models for Simulating Hydrologic and Water Quality Responses from an Urbanizing Watershed. ASAE Annual International Meeting Sponsored by ASAE Riviera Hotel and Convention Center Las Vegas, Nevada, USA, p. 27- 30, 2003.

MAGID, J.; TIESSEN, H.; CONDRON, L.M. Dynamics of organic phosphorus in soils under natural and agricultural ecosystems. In: PICCOLO, A., ed. Humic substances in terrestrial ecosystems. Amsterdan, Elsevier, p.429-466, 1996.

MARTINS FILHO, M.V. Modelagem do processo de erosão e padrão espacial da erodibilidade em entressulcos. Jaboticabal: FCAV, 2007. 121f. Tese (Livre-docência) Faculdade de Ciências Agrárias e Veterinárias, Universidade Estadual Paulista, Jaboticabal, 2007.

PEREIRA, S. B.; PRUSKI, F. F.; SILVA, D. D. da; MATOS, A. T. de. Desprendimento e arraste do solo pelo escoamento superficial. Revista brasileira de Engenharia Agrícola e Ambiental, v.7, p.423-429, 2003.

ROSOLEM, C.A.; PEREIRA, H.F.M.; BESSA, A.M.; AMARAL,P.G. Nitrogen in soil and cotton growth as affected by limingand $\mathrm{N}$ fertilizer. In: WRIGHT, R.J.; BALIGAR, V.C.;MURRMANN, R.P. (Ed.) Plant-soil interactions at low pH.Dordrecht: Kluwer, p.321-325, 1990.

SORATTO, R. P.; CARVALHO, M. A. C. de; ARF, O. Teor de clorofila e produtividade do feijoeiro em razão da adubação nitrogenada. Pesquisa Agropecuária Brasileira, Brasília, DF, v. 39, n. 9, p. 895-901, 2004.

SOUZA, R.F.; FAQUIM, V.; TORRES, P.R.F.; BALIZA, D.P. Calagem e adubação orgânica: influência na adsorção de fósforo em solos. Revista Brasileira de Ciência do Solo, Campinas, v.30, p.975-983, 2006

TRAINA, S.J.; SPOSITO, G.; HESTERBERG, D.; KAFKAFI, U. Effects of pH and organic acids on orthophosphate solubility in an acidic, montmorillonitic soil. Soil Science Society American Journal, Madison, v.50, p.45-52, 1986.

VEITH, T. L.; LIEW, M. W. VAN; BOSCH, D. D.; ARNOLD, J. G. Parameter sensitivity and uncertainty in SWAT: A comparison across five USDA-ARS watersheds. Transactions of the ASABE, v.53, p.1477-1486, 2010.

VIEUX, B. E. Distributed hydrologic modeling using GIS. 2.ed. Dordrecht: Kluwer Academic Publishers, 2004. 289p.

WHITE, R.E. Leaching. In: WILSON, J. R. Advances in nitrogen cycling in agricultural 
ecosystems. Wallinggord: C.A.B. International, p.193-211, 1987.

YAN, B.; FANG, N. F.; ZHANG, P. C.; SHI, Z. H. 2013. Impacts of land use change on watershed streamflow and sediment yield: an assessment using hydrologic modelling and partial least squares regression. Journal of Hydrology, v. 484, p. 26-37, 2013. 